\title{
The positive aspects of smoothness in Banach lattices
}

\author{
Marek Wójtowicz
}

Received: 23 October 2011 / Accepted: 6 February 2012 / Published online: 26 February 2012

C The Author(s) 2012. This article is published with open access at Springerlink.com

\begin{abstract}
Let $X$ be a Banach lattice, and let $x \in X \backslash\{0\}$. We study the structure of the set $\operatorname{Grad}(x)$, of all supporting functionals of $x$. If $X$ is a Dedekind $\sigma$-complete Banach lattice, there is an isometry from $\operatorname{Grad}(x)$ onto $\operatorname{Grad}(|x|)$; hence the elements $x$ and $|x|$ are smooth simultaneously. And if, additionally, $X^{*}$ is strictly monotone then $\operatorname{Grad}(|x|)$ consists of positive functionals. As a by-product of our results we obtain that an arbitrary Banach lattice $X$ is strictly monotone whenever its dual $X^{*}$ is smooth.
\end{abstract}

Keywords Smooth point - Strictly monotone Banach lattice · Orlicz space ·

Marcinkiewicz space $\cdot M$-ideal

Mathematics Subject Classification (2010) $\quad 46 \mathrm{~B} 20 \cdot 46 \mathrm{~B} 42$

\section{Introduction and notations}

We use standard notations and for notions undefined here we refer the reader to the monographs $[1,15]$.

Here and below $X$ denotes a fixed real Banach lattice, $X^{*}$ is its topological dual, and $S_{X}$ denotes the unit sphere of $X$. The ideal of order continuous elements of $X$ is denoted by $X_{a}$. For $x \in X \backslash\{0\}$ the symbol $\operatorname{Grad}(x)$ stands for the set of all supporting functionals of $x$, i.e., $\operatorname{Grad}(x)=\left\{f \in S_{X^{*}}: f(x)=\|x\|\right\}$. If $Y$ is a sublattice of $X$, or $X$ is a sublattice of $Y$, and $x \in Y$, then the $\operatorname{symbol} \operatorname{Grad}_{Y}(x)$ denotes the set $\left\{\phi \in S_{Y^{*}}: \phi(x)=\|x\|\right\}$, where $Y^{*}$ is the topological dual of $Y$. In this paper $Y$ will be either an ideal of $X$ or $Y=X^{* *}$.

M. Wójtowicz $(\varangle)$

Instytut Matematyki, Uniwersytet Kazimierza Wielkiego, Pl. Weyssenhoffa 11,

85-072 Bydgoszcz, Poland

e-mail: mwojt@ukw.edu.pl

Birkhäuser 
Let us recall that an element $x \in X \backslash\{0\}$ is said to be smooth if the $\operatorname{set} \operatorname{Grad}(x)$ is a singleton. Over the last 30 years, there have been published many papers devoted to smooth points in concrete Banach lattices (see, e.g., [4,7-9] and the references therein). Most of the proofs are too technical, and there is no general point of view both on the structure of $\operatorname{Grad}(x)$ and smooth points of $X$.

The purpose of this paper is to show that in typical cases (e.g., for the class of real Köthe spaces) the structures of the sets of $\operatorname{Grad}(x)$ and $\operatorname{Grad}(|x|)$ are "similar" (Theorem 1b). It is rather clear that a positive element $x$ is smooth if its (unique) supporting functional is positive (in Lemma 1 of Sect. 3 we prove a more general result). In this case $\operatorname{Grad}(x) \subset\left(X^{*}\right)^{+}$, but the latter inclusion also happens to be true if $X^{*}$ is strictly monotone (Theorem 2). In Theorem 3 we prove that, under a few natural assumptions about the order continuous part $X_{a}$ of $X$, an element $x \in X_{a}$ is smooth in $X_{a}$ iff it is smooth in $X$. Moreover, in Corollary 2 we show that the smoothness of $X^{*}$ implies the strict monotonicity of $X$.

Let us fix further notation. A functional $f \in X^{*}$ is said to be order continuous, if the condition $x_{\alpha} \downarrow 0$ in $X$ implies $|f|\left(x_{\alpha}\right) \rightarrow 0$. The band of order continuous elements of $X^{*}$ is denoted by $X_{n}^{*}$. If $W$ is a nonempty subset of $X$, the symbol $W^{\perp}$ denotes the annihilator (in $X^{*}$ ) of $W$.

A function $N:[0, \infty) \rightarrow[0, \infty)$ is said to be an Orlicz function if it is convex, continuous, with $N(0)=0$ and $N \neq \equiv 0$. Let $(\Omega, \Sigma, \mu)$ be a fixed $\sigma$-finite measure space, and let $L_{0}(\mu)$ denote the linear lattice of all (classes of) real $\mu$-measurable functions on $\Omega$. The function $N$ determines a functional $\rho_{N}: L_{0}(\mu) \rightarrow[0, \infty)$ defined by the rule $\rho_{N}(x)=\int_{\Omega} N(|x(\omega)|) d \mu$. The subspace

$$
L_{N}:=\left\{x \in L_{0}(\mu): \rho_{N}(\lambda x)<\infty \text { for some } \lambda>0\right\}
$$

is called an Orlicz space. It is a Dedekind complete Banach lattice with respect to the Luxemburg norm $\|x\|=\inf \left\{\lambda>0: \rho_{N}(x / \lambda) \leq 1\right\}$, and its order continuous part $\left(L_{N}(\mu)\right)_{a}$ equals $E_{N}:=\left\{x \in L_{0}(\mu): \rho(\lambda x)<\infty\right.$ for all $\left.\lambda>0\right\}$ (see, e.g., [3,14]).

\section{The results}

The following theorem allows us to reduce the studies of smoothness of arbitrary points of some Banach lattices to smoothness of their moduli. In particular, this is so in the class of Banach function lattices, e.g., Orlicz lattices.

Theorem 1 (a) Let $X$ be an arbitrary Banach lattice, and let $x \in X^{+} \backslash\{0\}$. If the element $x$ is smooth, then $\operatorname{Grad}(x)=\{f\}$, where $f \geq 0$.

(b) Let $X$ be a Dedekind $\sigma$-complete Banach lattice, and let an element $x=x^{+}-$ $x^{-} \in X$ be such that $x^{+}, x^{-} \neq 0$. Then there is a linear auto-isometry $\theta$ of $X$ such that its adjoint $\theta^{*}$ maps $\operatorname{Grad}(x)$ onto $\operatorname{Grad}(|x|)$. Hence, the elements $x$ and $|x|$ are smooth simultaneously.

For $x$ a positive element of $X$, we set

$$
\operatorname{Grad}(x)^{+}:=\left\{0 \leq f \in X^{*}:\|f\|=1, f(x)=\|x\|\right\}=\operatorname{Grad}(x) \cap\left(X^{*}\right)^{+} .
$$


Let us notice that, since $x$ is positive, its norm is attained by a positive element $f$ of $X^{*}$, and hence the set $\operatorname{Grad}(x)^{+}$is not empty.

We shall say that $X$ is positively smooth if every $x \in X^{+}$is smooth (by Theorem 1(a) we then also have $\left.\operatorname{Grad}(x)=\operatorname{Grad}(x)^{+}\right)$. From Theorem 1(b) we immediately obtain the following corollary.

Corollary 1 Let $X$ be a Dedekind $\sigma$-complete Banach lattice. Then $X$ is smooth if (and only if) it is positively smooth.

In other words, Corollary 1 says that, to check if a Dedekind $\sigma$-complete Banach lattice $X$ is smooth, it is enough to check if for every $x \in X^{+}$the set $\operatorname{Grad}(x)^{+}$is a singleton.

Kurc [10, p. 156] defines $X$ to be order smooth if, for every positive $x \in X$, the set $\operatorname{Grad}(x)^{+}$does not contain nontrivial order intervals. Hence, by Corollary 1 , for $X$ Dedekind $\sigma$-complete, we have

$$
\text { smooth } \Longleftrightarrow \text { positively smooth } \Rightarrow \text { order smooth. }
$$

Moreover, by [10, Theorem 1], if $X^{*}$ is order smooth, then $X$ is strictly monotone (i.e., the condition $0 \leq x<y$ in $X$ implies $\|x\|<\|y\|)$. Since $X^{*}$ is Dedekind complete, from $(*)$ we immediately obtain the following connection between smoothness and strict monotonicity.

Corollary 2 Let $X$ be a Banach lattice such that its dual $X^{*}$ is smooth. Then $X$ is strictly monotone. In particular, if $X$ is reflexive and smooth, then $X^{*}$ is strictly monotone.

Remark 1 The implications in the above corollary cannot be reversed, in general. In the non-reflexive case, the Banach lattice $X=\ell_{1}$ is strictly monotone, yet its dual $X^{*}=\ell_{\infty}$ is non-smooth.

For the reflexive-space case, let us consider the $\ell_{2}$-sum $X=\left(\sum_{n=1}^{\infty} X_{n}\right)_{2}$ of the two-dimensional Banach lattices $X_{n}=\ell_{\infty}^{(2)}, n=1,2, \ldots$, endowed with the supnorm. Then its dual $X^{*}=\left(\sum_{n=1}^{\infty} X_{n}^{*}\right)_{2}$ is strictly monotone, because every $X_{n}^{*}$ is lattice isometric to $\ell_{1}^{(2)}$, while $X$ is evidently non-smooth.

It should be clear that if $X$ is a Banach lattice and $x \in X^{+}$, then a supporting functional $f$ of $x$ need not be positive (the simplest example is $x=(0,1) \in X=\ell_{1}^{(2)}$ and $f=(1,-1) \in X^{*}=\ell_{\infty}^{(2)}$; cf. Lemma 1 in Sect. 3). But, by Theorem 1(a), if $x$ is additionally smooth then $\operatorname{Grad}(x) \subset\left(X^{*}\right)^{+}$. In the next theorem we present yet another sufficient condition for the latter inclusion to hold.

Theorem 2 Let $X$ be an arbitrary Banach lattice such that its dual $X^{*}$ is strictly monotone. Then, for every positive element $x$ of $X$, we have the equality

$$
\operatorname{Grad}(x)=\operatorname{Grad}(x)^{+}
$$


Remark 2 In the above theorem, $X$ is not necessarily Dedekind $\sigma$-complete, and hence we do not claim there is a connection between $\operatorname{Grad}(x)$ and $\operatorname{Grad}(|x|)$. However, if $\pi$ denotes the canonical (and order) embedding of $X$ into $X^{* *}$ then, by Theorem 1(b), there is an isometry from $\operatorname{Grad}_{X^{* *}}(\pi(x))$ onto $\operatorname{Grad}_{X^{* *}}(\pi(|x|))$.

Since the dual $X^{*}$ of every $A M$-space $X$ is an $A L$-space, $X^{*}$ is strictly monotone. Hence, by Theorem 2, we obtain the following corollary.

Corollary 3 Let $K$ be a completely regular topological space, and let $C_{b}(K)$ denote the real space of all continuous and bounded functions on $K$. Then every positive $x \in C_{b}(K)$ fulfils equality (**).

For the purpose of the next theorem, let us recall that a closed subspace $W$ of a Banach space $Y$ is an $M$-ideal [5], if there is a projection $P: Y^{*} \rightarrow Y^{*}$ with range $W^{\perp}$ such that $\left\|y^{*}\right\|=\left\|P y^{*}\right\|+\left\|(I-P) y^{*}\right\|$ for all $y^{*} \in Y^{*}$.

Theorem 3 Let $X$ be a real Banach lattice such that its order continuous part $X_{a}$ is order dense in $X$. If $X_{a}$ is an M-ideal then, for every element $x \in X_{a} \backslash\{0\}$, the set $\operatorname{Grad}(x)$ consists of order continuous functionals on $X$ :

$$
\operatorname{Grad}(x) \subset X_{n}^{*}
$$

Moreover, if the topological dual $X_{a}^{*}\left(\right.$ of $\left.X_{a}\right)$ is strictly monotone, then

$$
\operatorname{Grad}(|x|)=\operatorname{Grad}(|x|)^{+}, \text {for all } x \in X_{a},
$$

and there are isometries $T$ and $S$ mapping $\operatorname{Grad}(x)$ onto $\operatorname{Grad}(|x|)$ and $\operatorname{Grad}(x)$ onto $\operatorname{Grad}_{X_{a}}(x)$, respectively. In particular, the elements $x$ and $|x|$ are smooth points both of $X$ and $X_{a}$ simultaneously.

The above theorem has an interpretation in two classes of Banach lattices.

Let $N$ be an Orlicz function. It is known that $E_{N}$ is the order continuous part of $L_{N}$ and is order dense in $L_{N}$ (see [3, Theorem 1.25] and [14, p.145], respectively). Furthermore $E_{N}$ is an $M$-ideal in $L_{N}$ (see [3, Theorems 1.47 and 1.48]). Similarly, the order continuous part $(M(\Psi))_{a}$ of a Marcinkiewicz space $M(\Psi)$ has the same properties whenever $(M(\Psi))_{a} \neq\{0\}$ (see [6] for details, or [12, Lemma 1 and p. 266]).

Although the smooth points in Orlicz and Marcinkiewicz spaces have been already described [2,9], the corollary below adds a new information about their sets. Its proof follows immediately from Theorem 3 and the remarks following it.

Corollary 4 Let $N$ be a finite Orlicz function. An element $x \in E_{N}$ (as well as its modulus $|x|)$ is smooth in $E_{N}$ if and only if it is smooth in $L_{N}$.

Similarly, if $(M(\Psi))_{a} \neq\{0\}$, then an element $x \in(M(\Psi))_{a}$ is smooth in $(M(\Psi))_{a}$ and in $M(\Psi)$ simultaneously. 


\section{Proofs}

In the proofs we shall apply the following result. It shows that if an element $x$ is positive, then the set $\operatorname{Grad}(x)$ contains a positive functional.

Lemma 1 Let $x$ be a strictly positive element of $X$, and let $f$ be a supporting functional of $x:\|x\|=f(x)$ and $\|f\|=1$. Then the positive part $f^{+}$of $f$ is a supporting functional of $x$, too. More exactly:

(i) $1=\left\|f^{+}+f^{-}\right\|=\left\|f^{+}\right\|$and $f^{+}(x)=\|x\|$, whence

(ii) $f^{-}(x)=0$.

In particular, the condition $f^{-}=0$ (i.e., $f \geq 0$ ) holds if, either the dual lattice $X^{*}$ is strictly monotone or $x$ is strictly positive on $X^{*}$ (i.e., the condition $h>0$ for $0 \leq h \in X^{*}$ implies $\left.h(x)>0\right)$.

Remark 3 By [1, Theorem 4.85], an element $x \in X^{+}$is strictly positive on $X^{*}$ iff $x$ is a quasi-interior point, i.e., the ideal $A_{x}$ generated by $x$ is norm dense in $X$. It is well known [1, pp. 267-268] that every separable Banach lattice $X$ has strictly positive points on $X^{*}$.

Proof of Lemma 1. From $1=\|f\|=\||f|\|$ we obtain

$$
f^{+}(x) \leq f^{+}(x)+f^{-}(x)=|f|(x) \leq\|x\| .
$$

Moreover,

$$
\|x\|=f^{+}(x)-f^{-}(x) \leq f^{+}(x) \leq\|x\| .
$$

Thus, $\|x\|=f^{+}(x), f^{-}(x)=0$, and $\left\|f^{+}\right\| \leq\|f\|=1$, whence

$$
\left\|f^{+}\right\|=1=\|f\|=\||f|\|=\left\|f^{+}+f^{-}\right\| .
$$

This proves parts $(i)$ and $(i i)$ of the lemma.

Now, if $X^{*}$ is strictly monotone then the inequality $f^{+} \leq f^{+}+f^{-}$and condition (1) imply that $f^{+}=f^{+}+f^{-}$. Hence $f^{-}=0$. The second particular case is obvious.

The following result is known to specialists in Banach lattices. We include its proof for the convenience of the reader.

Lemma 2 Let $X$ be a Banach lattice such that its order continuous part $X_{a}$ is order dense in $X$, and let $X_{a}^{*}$ denote the topological dual of $X_{a}$. Then:

(i) The Banach lattices $X_{n}^{*}$ and $X_{a}^{*}$ are order isometric: the isometry from $X_{n}^{*}$ onto $X_{a}^{*}$ is of the form: $r(f)=f_{\mid X_{a}}$.

(ii) Every element $f \in X^{*}$ has the form $f=f_{n}+f_{s}$, where $f_{n}$ is order continuous and $f_{s}$ vanishes on $X_{a}$, i.e., $X^{*}=X_{n}^{*}+\left(X_{a}\right)^{\perp}$. 
Proof of Lemma 2. (i) By [13, Corollary 1.2], every $\varphi \in X_{a}^{*}$ has a unique and norm-preserved extension $e(\varphi)$ to an element of $X_{n}^{*}$. Let $f \in X_{n}^{*}$ be fixed, and set $\varphi:=f_{\mid X_{a}}$. Then $\varphi \in X_{a}^{*}$. By the uniqueness of the extension $e$, we have $e(\varphi)=f$; moreover, $\|\varphi\| \leq\|f\|$. We must also have $\|\varphi\|=\|f\|$ since, otherwise, $\|f\|=\|e(\varphi)\|<\|f\|$, a contradiction. Hence, $\|r(f)\|=\|\varphi\|=$ $\|f\|$, as claimed.

(ii) We have $X^{*}=X_{n}^{*}+X_{s}^{*}$, where $X_{s}^{*}$ is the disjoint complement of the band $X_{n}^{*}$. Since $X_{a}$ is order dense in $X,\left[15\right.$, Theorem 88.11] implies that $X_{s}^{*}=\left(X_{a}\right)^{\perp}$.

Proof of Theorem 1. Part (a) follows immediately from Lemma 1.

For the proof of part (b), let $P$ denote the order projection onto the principal band $\left(x^{+}\right)^{d d}$. Set $\theta:=P-P^{c}$, where $P^{c}=I-P$ and $I$ is the identity on $X$. Since $x^{-} \wedge x^{+}=0$, we have that $x^{-} \in\left(x^{+}\right)^{d}=P^{c}(X)$, and so $P\left(x^{-}\right)=0$ and $P\left(x^{+}\right)=$ $x^{+}$; similarly, $P^{c}\left(x^{+}\right)=0$ and $P^{c}\left(x^{-}\right)=x^{-}$. Hence,

$$
\theta(x)=\left(P-P^{c}\right)\left(x^{+}-x^{-}\right)=x^{+}+x^{-}=|x| ; \text { similarly, } \theta(|x|)=x .
$$

Since for every $y \in X$ the elements $P(y)$ and $P^{c}(y)$ are disjoint, we obtain (see [1, Ex. 3, p. 21])

$$
|\theta(y)|=\left|P(y)-P^{c}(y)\right|=\left|P(y)+P^{c}(y)\right|=|y|,
$$

whence $\|\theta(y)\|=\||\theta(y)|\|=\||y|\|=\|y\|$. Thus, $\theta$ is an isometry of $X$, and $\theta$ maps $X$ onto $X$ because it is an involution, i.e., $\theta^{2}=I$. Thus, $\theta^{*}$ is an auto-isometry of $X^{*}$.

Now let $f \in \operatorname{Grad}(x): f(x)=\|x\|$ and $\|f\|=1$. Then, by (2), we obtain

$$
\theta^{*}(f)(|x|)=f(\theta(|x|))=f(x)=\|x\|=\||x|\| ;
$$

thus $\theta^{*}(f) \in \operatorname{Grad}(|x|)$ because $\theta^{*}$ is an isometry. Hence, $\theta^{*}(\operatorname{Grad}(x)) \subset \operatorname{Grad}(|x|)$. Similarly, by (2), we obtain the reversed inclusion $\operatorname{Grad}(|x|) \subset \theta^{*}(\operatorname{Grad}(x))$.

Proof of Theorem 2. We have to prove the nontrivial inclusion $\operatorname{Grad}(x) \subset \operatorname{Grad}(x)^{+}$. Since $x \geq 0$, from the second part of Lemma 1 we obtain that every element of $\operatorname{Grad}(x)$ is positive, hence it is an element of $\operatorname{Grad}(x)^{+}$. That is, $\operatorname{Grad}(x) \subset \operatorname{Grad}(x)^{+}$, as claimed.

Proof of Theorem 3. Let $x \in X_{a} \backslash\{0\}$, and let $f \in \operatorname{Grad}(x)$. By Lemma 2 (ii), $f=$ $f_{n}+f_{s}$, where $f_{s \mid X_{a}}=0$ and $f_{n} \in X_{n}^{*}$. Hence

$$
\|x\|=f(x)=f_{n}(x) .
$$

Since $X_{a}$ is an $M$-ideal,

$$
1=\|f\|=\left\|f_{n}\right\|+\left\|f_{s}\right\|
$$

Thus from (3) and (4) we obtain $\left\|f_{n}\right\|=1$ and $f_{s}=0$, whence $f=f_{n} \in X_{n}^{*}$. So that, $\operatorname{Grad}(x) \subset X_{n}^{*}$, as claimed. 
By Lemma 2 (i), there is an order isometry $r$ from $X_{n}^{*}$ onto $X_{a}^{*}$. For $x \in X_{a}$, set

$$
\operatorname{Grad}_{X_{a}}(x):=\left\{\varphi \in X_{a}^{*}: \varphi(x)=\|x\|,\|\varphi\|=1\right\} .
$$

From the form of $r$ we easily obtain that $r$ maps $\operatorname{Grad}(x)$ onto $\operatorname{Grad}_{X_{a}}(x)$. Moreover, by Theorem 1 , there is an auto-isometry $\theta$ of $X_{a}$ such that its adjoint, $\theta^{*}, \operatorname{maps}_{\operatorname{Grad}_{X_{a}}}(x)$ onto $\operatorname{Grad}_{X_{a}}\left(|x|_{X_{a}}\right)$, where $|x|_{X_{a}}$ denotes the modulus of $x$ in the (sub)lattice $X_{a}$. But since $X_{a}$ is an ideal of $X$, the moduli $|x|_{X_{a}}$ and $|x|$ coincide. Hence, the composition $S:=\theta^{*} r$ is an isometry mapping $\operatorname{Grad}(x)$ onto $\operatorname{Grad}_{X_{a}}(|x|)$, and so $T:=r^{-1} S$ maps isometrically $\operatorname{Grad}(x)$ onto $\operatorname{Grad}(|x|)$. In particular, the elements $x$ and $|x|$ are smooth (both in $X_{a}$ and in $X$ ) simultaneously.

Finally, Theorem 2 implies that $\operatorname{Grad}_{X_{a}}(|x|) \subset\left(X_{a}^{*}\right)^{+}$, whence, by $(* * *)$,

$$
\operatorname{Grad}(|x|)=r^{-1}\left(\operatorname{Grad}_{X_{a}}(|x|)\right) \subset\left(X_{n}^{*}\right)^{+} \subset\left(X^{*}\right)^{+} .
$$

By definition, $\operatorname{Grad}(|x|)=\operatorname{Grad}(|x|)^{+}$.

The proof of Theorem 3 is complete.

Open Access This article is distributed under the terms of the Creative Commons Attribution License which permits any use, distribution, and reproduction in any medium, provided the original author(s) and the source are credited.

\section{References}

1. Aliprantis, C.D., Burkinshaw, O.: Positive Operators. Academic Press, New York (1985)

2. Chen, S.: Smoothness of Orlicz spaces. Comment. Math. Prace. Mat. 27, 49-58 (1987)

3. Chen, S.: Geometry of Orlicz spaces, Dissertationes Math. 361 (1996)

4. Bian, S., Hudzik, H., Wang, T.: Smooth, very smooth and strongly smooth points in Musielak-Orlicz sequence spaces. Bull. Aust. Math. Soc. 63, 441-457 (2001)

5. Harmand, P., Werner, D., Werner, W.: $M$-ideals in Banach Spaces and Banach Algebras. Springer, Berlin (1993)

6. Kamińska, A., Lee, H.J.: $M$-ideal properties in Marcinkiewicz spaces. Ann. Soc. Math. Pol. Ser. I. Comment. Math. (Special Issue) 123-144 (2004)

7. Kamińska, A., Lee, H.J., Lewicki, G.: Extreme and smooth points in Lorentz and Marcinkiewicz spaces with applications to contractive projections. Rocky Mountain J. Math. 39, 1533-1572 (2009)

8. Kaminska, A., Parrish, A.M.: Note on extreme points in Marcinkiewicz function spaces. Banach J. Math. Anal. 4, 1-12 (2010)

9. Kamińska, A., Parrish, A.M.: Smooth points in Marcinkiewicz function spaces. J. Convex Anal. 18, 379-390 (2011)

10. Kurc, W.: A dual property to uniform monotonicity for Banach lattices. Collect. Math 44, 155-165 (1993)

11. Luxemburg, W.A.J., Zaanen, A.C.: Riesz Spaces I. North-Holland, Amsterdam (1971)

12. Plichko, A., Wójtowicz, M.: Copies of $c_{0}(\Gamma)$ and $\ell_{\infty}(\Gamma) / c_{0}(\Gamma)$ in quotients of Banach spaces with applications to Orlicz and Marcinkiewicz spaces. Indag. Math. N.S. 18, 251-268 (2007)

13. Schaefer, H.H., Zhang, X-D.: Extension properties of order continuous functionals and applications to the theory of Banach lattices. Indag. Math. N.S. 5, 107-118 (1994)

14. Wnuk, W.: On the order-topological properties of the quotient space $L / L_{A}$. Studia Math. 79, 139-149 (1984)

15. Zaanen, A.C.: Riesz Spaces II. North-Holland, Amsterdam (1980) 